\title{
Tissue microarray analysis of human FRATI expression and its correlation with the subcellular localisation of $\beta$-catenin in ovarian
}

\section{tumours}

\author{
Y Wang', SM Hewitt', S Liu', X Zhou', H Zhu', C Zhou', G Zhang', L Quan', J Bai' and N Xu*,I \\ 'Laboratory of Cell and Molecular Biology, Cancer Institute \& Cancer Hospital, Chinese Academy of Medical Sciences \& Peking Union Medical College, \\ Beijing 10002 I, People's Republic of China; ${ }^{2}$ Tissue Array Research Program, Laboratory of Pathology, Center for Cancer Research, National Cancer \\ Institute, National Institutes of Health, Bethesda, MD 20892-4605, USA
}

The mechanisms involved in the pathogenesis of ovarian cancer are poorly understood, but evidence suggests that aberrant activation of Wnt/ $\beta$-catenin signalling pathway plays a significant role in this malignancy. However, the molecular defects that contribute to the activation of this pathway have not been elucidated. Frequently rearranged in advanced T-cell lymphomas-I (FRATI) is a candidate for the regulation of cytoplasmic $\beta$-catenin. In this study, we developed in situ hybridisation probes to evaluate the presence of FRATI and used an anti- $\beta$-catenin antibody to evaluate by immunohistochemistry the expression levels and subcellular localisation of $\beta$-catenin in ovarian cancer tissue microarrays. Expression of FRATI was found in some human normal tissues and $47 \%$ of ovarian adenocarcinomas. A total of $46 \%$ of ovarian serous adenocarcinomas were positive for FRATI expression. Accumulation of $\beta$-catenin in the nucleus and/or cytoplasm was observed in 55\% ovarian adenocarcinomas and in 59\% of serous adenocarcinomas. A significant association was observed in ovarian serous adenocarcinomas between FRATI and $\beta$-catenin expression $(P<0.0 \mathrm{I})$. These findings support that $\mathrm{Wnt} / \beta$-catenin signalling may be aberrantly activated through FRATI overexpression in ovarian serous adenocarcinomas. The mechanism behind the overexpression of FRATI in ovarian serous adenocarcinomas and its significance is yet to be investigated. British Journal of Cancer (2006) 94, 686-69I. doi:I0.I038/sj.bjc.6602988 www.bjcancer.com

Published online 14 February 2006

(c) 2006 Cancer Research UK

Keywords: FRATI; $\beta$-catenin; Wnt pathway; ovarian cancer; tissue microarray

Ovarian cancer is the sixth most common cancer in women worldwide and a highly aggressive gynaecological malignancy producing almost 125000 deaths yearly (Parkin et al, 2005). Despite advances in detection and cytotoxic therapies, only $30 \%$ of patients with advanced-stage ovarian cancer survive 5 years after initial diagnosis (Parkin et al, 2005). Therefore, an understanding of the molecular mechanisms involved in ovarian cancer pathogenesis and progression has the potential to have a significant impact on the outcomes for this devastating disease. Most ovarian neoplasms are adenocarcinomas that occur as four major histological subtypes, serous, mucinous, endometrioid and clear cell, with serous being the most common. Current data indicate that each of these histological subtypes is associated with distinct morphologic and molecular genetic alterations (Bell, 2005). Further investigations of the molecular mechanisms promoting ovarian cancer are necessary to determine how each of the subtypes emerge. Relatively little is known about the molecular events that lead to the development of ovarian cancer.

Recently, accumulation of nuclear and/or cytoplasmic $\beta$-catenin has been observed in ovarian cancer (Gamallo et al, 1999; Wright et al, 1999; Rask et al, 2003; Kildal et al, 2005). $\beta$-Catenin is a multifunctional protein involved in at least two important

*Correspondence: Dr N Xu; E-mail: xningzhi@public.bta.net.cn Received 13 October 2005; revised 9 January 2006; accepted 17 January 2006; published online 14 February 2006 biological processes: cell-cell adhesion and $\mathrm{Wnt} / \beta$-catenin signalling pathway (Nelson and Nusse, 2004). It is believed that accumulation of $\beta$-catenin in the cytoplasm favours its translocation to the nucleus as a cofactor for transcription factors of the T-cell factor/lymphoid enhancing factor (TCF/LEF) family and activates the transcription of $\mathrm{Wnt} / \beta$-catenin target genes, which are involved in cellular differentiation and proliferation (Giles et al, 2003). Although compelling evidence has indicated a critical role for signalling by $\beta$-catenin in the tumorigenesis of ovarian cancer, mutations in the $\beta$-catenin gene are infrequent in ovarian carcinoma and interestingly only described in the endometrioid type of epithelial ovarian tumours (Gamallo et al, 1999; Wright et al, 1999; Ueda et al, 2001; Giles et al, 2003). Mutations of adenomatous polyposis coli (APC) or Axin occur rarely in ovarian cancer (Giles et al, 2003). Therefore, additional mechanisms can be used to upregulate $\beta$-catenin levels in other types of ovarian tumours. Previous studies have shown that frequently rearranged in advanced T-cell lymphomas-1 (FRAT1) is strikingly overexpressed in some human cancer cells (Saitoh and Katoh, 2001; Saitoh et al, 2002). Furthermore, FRAT1 is a positive regulator of the $\mathrm{Wnt} / \beta$-catenin pathway, which can inhibit glycogen synthase kinase 3 (GSK-3) activity towards $\beta$-catenin, at least in part, by preventing Axin binding to GSK-3 (Ferkey and Kimelman, 2002; Fraser et al, 2002; Giles et al, 2003; van Amerongen and Berns, 2005). However, whereas FRAT1 is a candidate for the regulation of cytoplasmic $\beta$-catenin, little is known with regard to the 
molecular relationship between FRAT1 and $\beta$-catenin in ovarian cancer.

In this study, we developed in situ hybridisation probes to evaluate the presence of FRAT1 and used an anti- $\beta$-catenin antibody to evaluate by immunohistochemistry the expression levels and subcellular localisation of $\beta$-catenin in formalin-fixed, paraffin-embedded human normal tissues and ovarian tumour tissues. This study was also aided by the use of tissue microarrays, allowing a much larger cohort of patient samples to be evaluated in a more standardised and rapid fashion. We demonstrate here that FRAT1 is expressed in some human normal tissues and overexpressed in human ovarian cancer. There is an obvious correlation between FRAT1 overexpression and aberrant nuclear and/or cytoplasmic accumulation of $\beta$-catenin in serous adenocarcinoma of ovary. Wnt/ $\beta$-catenin signalling may be aberrantly activated through FRAT1 overexpression in ovarian serous adenocarcinomas.

\section{MATERIALS AND METHODS}

\section{Construction of tissue arrays}

TARP5-T-BO-1 tumour tissue arrays were constructed by the NCI TARP Lab using anonymous donor blocks obtained from the Cooperative Human Tissue Network. Detailed information about TARP5-T-BO-1 tumour tissue arrays is available at www.cancer. gov/tarp. The arrays were produced as described previously (Hewitt, 2004), using a manual tissue arrayer (Beecher Instruments, Silver Spring). The array design included specimens of 75 ovarian malignancies of surface epithelial origin, 65 breast carcinomas and 35 cores of normal tissues representing 17 different histologies. Sections $(5 \mu \mathrm{m})$ were cut from the array blocks using tape sectioning materials from Instrumedics (Hackensack, NJ, USA).

\section{Plasmids construction}

The FRAT1 expression vector was constructed by cloning the open reading frame of the human FRAT1 cDNA obtained from HEK293 cells into pcDNA3 vector (Invitrogen, CA, USA). Correct cloning of FRAT1 cDNA fragments was confirmed by sequencing. The resulting plasmid was designated FRAT1/pcDNA3. Probes for in situ hybridisation were generated by in vitro transcription using SP6 polymerase (Roche, Germany) as described below.

\section{In situ hybridisation}

For in situ hybridisation, digoxigenine (DIG)-labelled FRAT1 probes were prepared using a DIG-RNA-labelling kit (Roche), according to the manufacturer's instructions. In brief, FRAT1/ pcDNA3 plasmids were linearised with HindIII and DIG-labelled probes were generated by in vitro transcription using SP6 polymerase (Roche). The amount of transcripts was monitored by $2 \%$ agarose gel electrophoresis and determined by spectrophotometry. Labelling efficiency was controlled by dot blot analysis of serial probe dilutions.

In situ hybridisation was performed according to the method described previously (Zhou et al, 2005). In brief, tissue sections were deparaffinised, rehydrated in serial dilutions of ethanol, and postfixed in $4 \%$ Tris-buffered saline (TBS)-buffered paraformaldehyde. Samples were treated with $0.2 \mathrm{moll}^{-1} \mathrm{HCl}$ for $10 \mathrm{~min}$ at room temperature and then permeabilised using proteinase $\mathrm{K}$ $\left(100 \mathrm{mg} \mathrm{l}^{-1}\right)$ at $37^{\circ} \mathrm{C}$ for $15 \mathrm{~min}$. Digestion was stopped by washing the samples in phosphate-buffered saline (PBS) ( $\mathrm{pH}$ 7.4). The samples were then dehydrated in serial dilutions of ethanol. DIGlabelled FRAT1 probes were diluted in hybridisation buffer $(50 \%$ formamide, $4 \times$ SSC, $5 \%$ dextran sulphate, $5 \times$ Denhardt's solution and $200 \mathrm{mg} \mathrm{ml}^{-1}$ denatured salmon sperm DNA). After probes were applied, the samples were covered with sterile coverslips. Hybridisation was performed overnight at $42^{\circ} \mathrm{C}$ in a sealed humidified chamber containing $50 \%$ formamide. Nonspecific binding or unbound probes were removed by the following posthybridisation washes: $2 \times$ SSC at room temperature $(2 \times 10 \mathrm{~min}), 1 \times \mathrm{SSC}$ at room temperature $(2 \times 10 \mathrm{~min})$, and finally, the sections were washed in TBS containing $0.1 \%$ Tween20 (TTBS). Hybridisation signals were detected using an alkaline phosphatase (AP)-conjugated anti-DIG antibody (Roche). After washing in TTBS, the slides were incubated with nitro blue tetrazolium/5-bromo-4-chloroindol-3-yl phosphate (NBT/BCIP) (Roche) for $10 \mathrm{~min}$. The FRAT1-positive cells were purple-blue stained. The negative controls were incubated with hybridising solution without FRAT1 probes.

\section{Immunohistochemistry}

Immunohistochemistry was performed using an UltraSensitive ${ }^{\mathrm{TM}}$ Kit (Maixin-Bio) according to the manufacturer's protocol as described previously (Wang et al, 2005). In brief, tissue sections were deparaffinised, and rehydrated in serial dilutions of ethanol. Endogenous peroxidase activity was blocked with 10-min incubation in a $3 \%$ hydrogen peroxide in PBS. Antigen retrieval was performed by boiling in citrate buffer $(0.01 \mathrm{M}, \mathrm{pH}=6.0)$ in a microwave oven. To reduce background nonspecific staining, slides were incubated with normal nonimmune serum for $30 \mathrm{~min}$. The excess serum was removed and slides were incubated for 60 min with anti- $\beta$-catenin (C19220; $1: 50$; Transduction Lab, KY, USA). Each incubation step was carried out at room temperature and was followed by three sequential washes ( 5 min each) in PBS. Slides were then incubated with biotin-conjugated second antibody for $30 \mathrm{~min}$, followed by incubation with streptavidinperoxidase for $30 \mathrm{~min}$. Peroxidase activity was identified by applying 3,3'-diaminobenzidine tetrachloride (DAB). Sections were rinsed in water and counterstained with haematoxylin. Finally, slides were dehydrated in alcohol, cleared in xylene. Slides were also stained in the absence of primary antibody to evaluate nonspecific secondary antibody reactions.

\section{Evaluation of staining}

All in situ hybridisation and immunostaining experiments were assessed by two experienced pathologists who were blinded to the origin of the sections. The membranous, cytoplasmic and nuclear staining was determined separately for each specimen. The staining intensity was graded as follows: 0 , no staining; 1 , weak staining; 2 , moderate staining and 3 , intense staining. Owing to too few cells or core missing in some sample cores, only 60 ovarian samples out of 75 cores and 12 different normal tissue samples in this tissue microarray were interpretable. For specimens that were uninterpretable, a score of NA was given. The data from breast samples are not shown here. In each case, the staining was scored as an average throughout the spot. FRAT1 expression within the tumour tissue was evaluated and categorised according to the staining intensity. Tumours were then further grouped into low (score 0) and high (scores 1-3) expression of FRAT1. Meanwhile, $\beta$-catenin protein expression profiles in cancer tissues were further grouped into cytoplasm/nucleus accumulation or no accumulation.

\section{Statistical analysis}

SPSS for Windows (SPSS Inc.) was used for statistical analysis. Correlation between the FRAT1 expression levels and $\beta$-catenin expression profiles on a per case basis was further analysed by Spearman's $\rho$ correlation coefficient test. Values of $P<0.05$ were considered statistically significant. 


\section{RESULTS}

\section{Site of reaction products}

The product of in situ hybridisation with the probes directed at FRAT1 was located in the cytoplasm of the cells (Figure 1A). Meanwhile, $\beta$-catenin immunostaining demonstrated two distinct staining patterns: one that was predominantly cytoplasmic, sometimes with concomitant nuclear staining and reduced membranous staining (Figure 1C); and one that was with no cytoplasmic or nuclear staining, often with membranous staining (Figure 1D).

\section{Expression of FRAT1 in human normal tissues}

The tissue microarrays containing various normal tissues and tumour tissues were hybridised with human DIG-labelled FRAT1 probes. The probes span the $\mathrm{C}$-terminal coding region, minimising the potential for crossreactivity between human FRAT1 and FRAT2. Analysis of the tissue microarrays revealed that FRAT1 was expressed in human endometrium, testis, pancreas and prostate (Figure 2). Expression of FRAT1 in other human normal tissues, including cerebellum, colon, kidney, liver, lung, salivary, spleen and thyroid, was not detected (Figure 2). These results were consistent with a previous report (Freemantle et al, 2002).

\section{Expression of FRAT1 and $\beta$-catenin in human ovarian tumour tissues}

FRAT1 was found in 28 of $60(46 \%)$ ovarian adenocarcinomas investigated. In total, $46 \%$ (19 out of 41 ) of all ovarian serous adenocarcinomas were positive for FRAT1 expression, followed by four out of 11 clear cell, four out of five endometrioid and one out
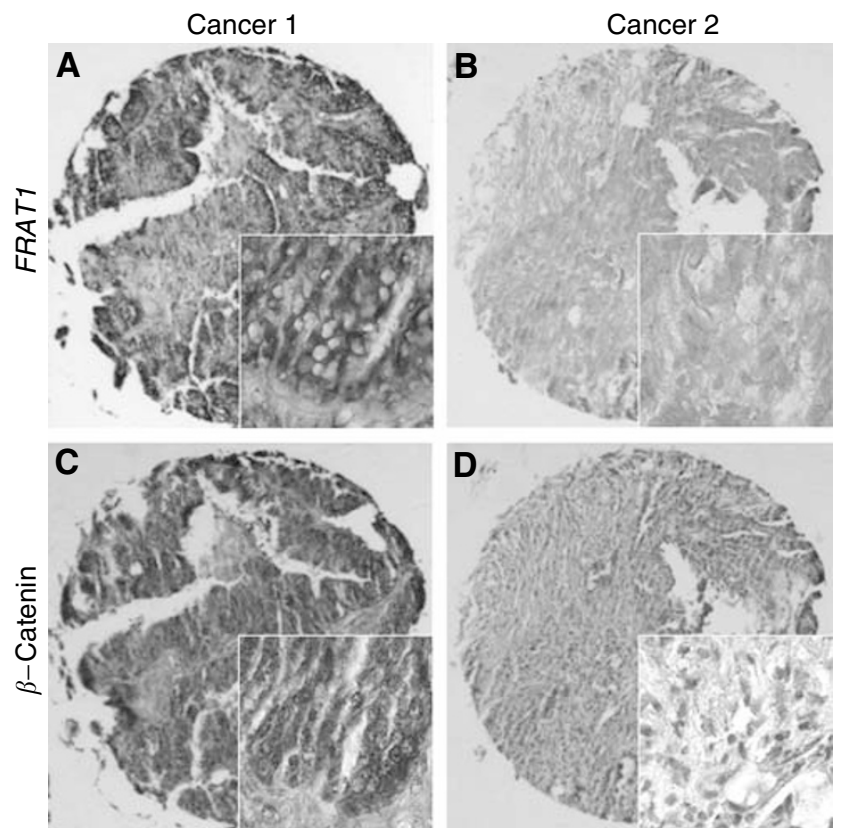

Figure I Correlation between FRATI overexpression and $\beta$-catenin localisation in human serous adenocarcinomas of ovary. Tissue microarrays of paraffin-embedded ovarian adenocarcinomas were stained with in situ hybridisation probes against FRATI or anti- $\beta$-catenin antibody and visualised by NBT/BCIP or DAB staining, respectively. Left panels, a representative high-FRATI staining specimen $(\mathbf{A})$ with accumulation of $\beta$-catenin in the cytoplasm and nucleus $(\mathbf{C})$; right panels, a representative specimen with Iow-FRATI staining (B) with no accumulation of $\beta$-catenin (D). Figures are $\times 100$, and inserts are $\times 400$. of three mucinous adenocarcinomas (Table 1). All analysed ovarian tumours showed membrane $\beta$-catenin expression with variable extension and intensity. Accumulation of $\beta$-catenin in the nucleus and/or cytoplasm was observed in 33 of 60 (55\%) ovarian adenocarcinomas. In serous adenocarcinomas, the percentage of cases with $\beta$-catenin accumulation was approximately $59 \%$. Nuclear and/or cytoplasmic expression of $\beta$-catenin was also observed in four out of 11 clear cell, five out of five endometrioid and zero out of three mucinous adenocarcinomas (Table 1).

\section{Correlation between subcellular localisation of $\beta$-catenin and overexpression of FRAT1 in ovarian serous adenocarcinomas}

To examine whether FRAT1 expression is correlated with increased expression and cytoplasmic/nuclear localisation of $\beta$-catenin in primary ovarian serous adenocarcinoma tissues, we compare the expression of FRAT1 and the subcellular localisation of $\beta$-catenin in 41 primary human ovarian serous adenocarcinoma tissues by in situ hybridisation and immunohistochemical staining, respectively. Previous studies demonstrated that $\beta$-catenin has an aberrant expression pattern in ovarian serous adenocarcinomas (Rask et al, 2003; Kildal et al, 2005). Approximately 59\% (24 out of 41) of ovarian serous adenocarcinoma samples showed cytoplasmic/nuclear accumulation accompanied by reduction of $\beta$-catenin localisation at membranes. More importantly, in most cases, $\beta$-catenin accumulation was correlated with high levels of FRAT1 expression in tumour specimens, whereas tumour tissues showing no accumulation of $\beta$-catenin generally contained relatively low levels of FRAT1 (Figure 1; Table 2). In 41 ovarian serous adenocarcinoma tissues examined, there was a significant correlation between FRAT1 and $\beta$-catenin expression, as determined by the Spearman rank correlation test $(P<0.01)$. These results suggest that FRAT1 may modulate the $\mathrm{Wnt} / \beta$-catenin pathway in ovarian serous adenocarcinomas.

\section{DISCUSSION}

Ovarian cancer is one of the most complex malignancies with a diversity of histological subtypes, including serous, clear cell, mucinous and endometrioid (Bell, 2005). Limited information is available as for the molecular alterations in ovarian cancer. The reports about the expression of $\beta$-catenin in ovarian cancer are a little confusing. However, it is consistent that nuclear $\beta$-catenin is almost exclusively present in endometriod type of ovarian cancer (Gamallo et al, 1999; Wright et al, 1999; Kildal et al, 2005). In other types of ovarian cancer, including serous type, the predominant aberrant expression pattern of $\beta$-catenin was cytoplasmic staining without nuclear accumulation (Rask et al, 2003; Kildal et al, 2005). These confusing phenomena may indicate and also may be due to distinct molecular genetic alterations in different types of ovarian caner (Bell, 2005). Although accumulation of cytoplasmic and nuclear $\beta$-catenin has been observed in serous ovarian cancer (Rask et al, 2003; Kildal et al, 2005), mutations of $\beta$-catenin in this subtype occur rarely (Gamallo et al, 1999; Wright et al, 1999; Ueda et al, 2001; Giles et al, 2003). The mechanism of accumulation of $\beta$ catenin in ovarian serous adenocarcinomas might be independent of genetic alterations of the $\beta$-catenin as shown in other tumours (Giles et al, 2003). Further mechanisms to stabilise $\beta$-catenin protein seem to be hidden in this subtype of ovarian cancer. It has been suggested that downregulated expression of APC (Karbova et al, 2002) or secreted frizzled-related proteins (SFRPs) inactivation by methylation (Takada et al, 2004) could explain, at least partially, why ovarian serous adenocarcinomas show increased $\beta$-catenin protein expression in spite of the absence of gene mutation. However, none of these studies has shown any statistical correlation between these molecules and subcellular localisation of 

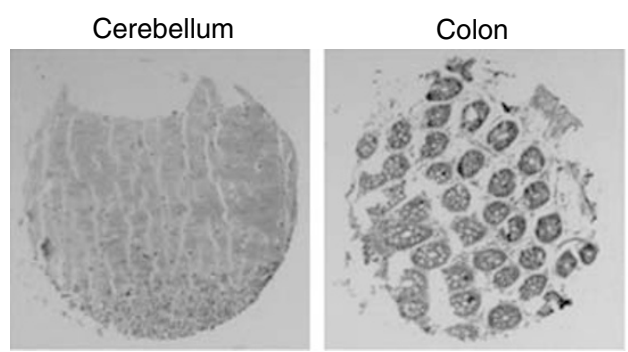

Lung
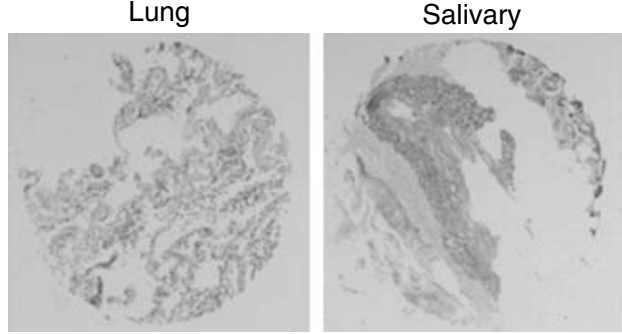

Endometrium

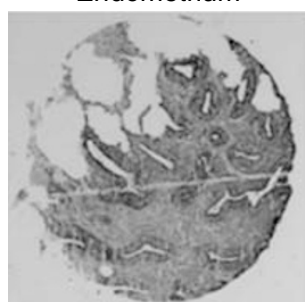

Testis

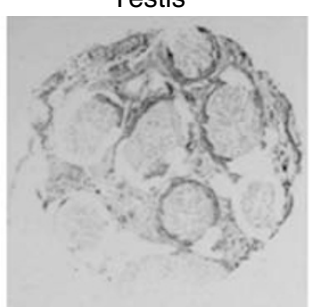

Kidney

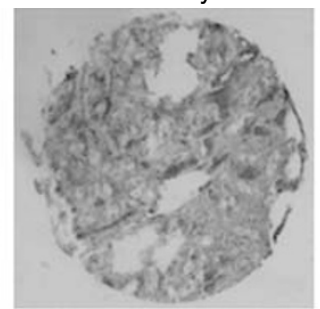

Spleen

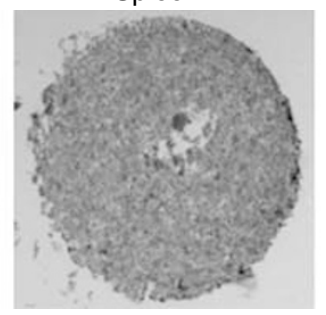

Pancreas

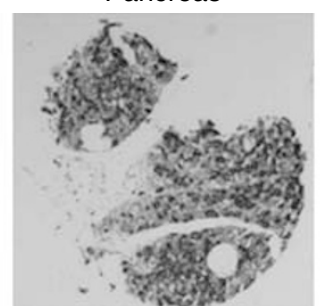

Liver

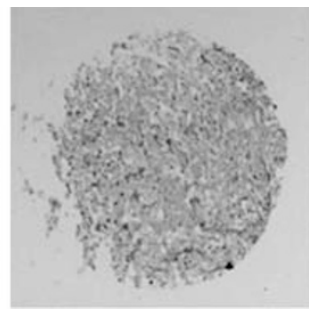

Thyroid

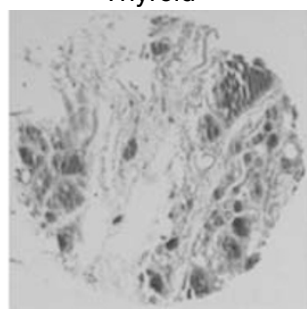

Prostate

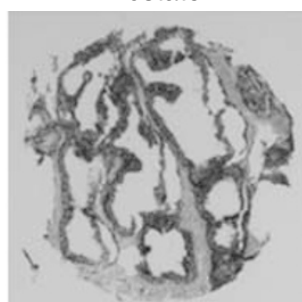

Figure 2 Expression of FRATI in human normal tissues. The tissue microarrays containing various normal tissues were hybridised with human DIGlabelled FRATI probes. Expression of FRATI was not detected in human cerebellum, colon, kidney, liver, lung, salivary, spleen and thyroid; meanwhile, there was FRATI expression in human endometrium, testis, pancreas and prostate.

Table I Association of FRATI and $\beta$-catenin expression with histological type in ovarian adenocarcinoma

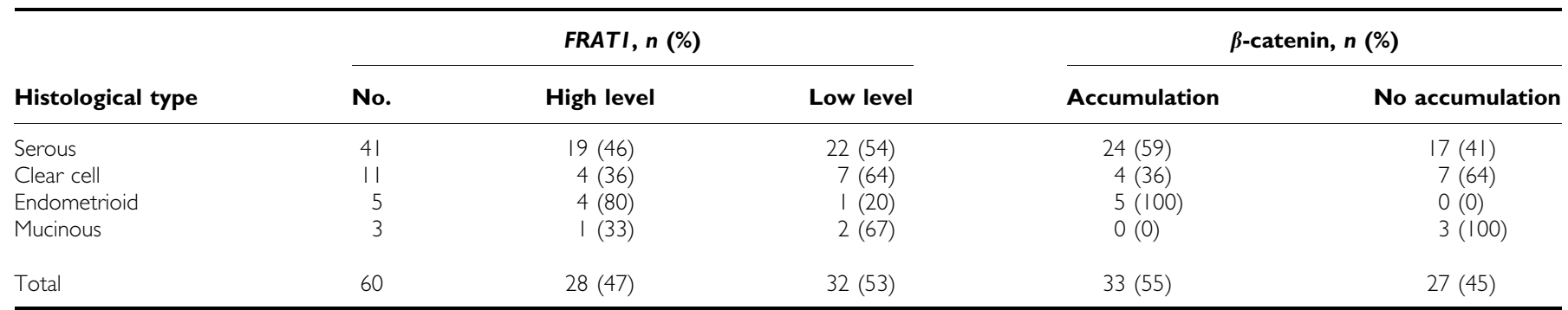

Table 2 Comparison of FRATI and $\beta$-catenin expression in serous adenocarcinoma of ovary

\begin{tabular}{lccc}
\hline & & FRATI expression & \\
\cline { 2 - 4 } $\boldsymbol{\beta}$-Catenin staining & Low level $(\mathbf{n}=\mathbf{2 2})$ & High level $(\boldsymbol{n}=\mathbf{1 9 )}$ & Total \\
\hline Accumulation & $7(17 \%)$ & $17(41 \%)$ & $24(59 \%)$ \\
No accumulation & $15(37 \%)$ & $2(5 \%)$ & $17(41 \%)$ \\
Total & $22(54 \%)$ & $19(46 \%)$ & $41(100 \%)$ \\
\hline
\end{tabular}

The level of FRATI expression and localisation of $\beta$-catenin were determined in 41 surgical specimens of serous adenocarcinoma of ovary, as shown in Figure I. The correlation was analysed using a Spearman rank correlation test, $P<0.01$.

$\beta$-catenin in tissue samples (Karbova et al, 2002; Takada et al, 2004). Whether deregulation of these molecules contributes to the accumulation of $\beta$-catenin in ovarian serous adenocarcinomas is yet to be established.
FRAT1 gene, mapping to human chromosome 10q24.1, is a human homologue of mouse proto-oncogene Frat1 (Jonkers et al, 1997; Saitoh and Katoh, 2001). Frat1 was initially cloned in a screen to identify genes that can accelerate lymphomagenesis in 
Moloney murine leukaemia virus (M-MuLV)-infected Eì-Pim1 or $\mathrm{H} 2-\mathrm{K}-\mathrm{Myc}$ transgenic mice (Jonkers et al, 1997). Its biological function remained unknown until its Xenopus homologue GSK-3binding protein (GBP) was identified as a GSK-3-binding protein (Yost et al, 1998). GSK-3-binding protein is required to specify the dorsal-ventral axis in Xenopus embryos. It appears to inhibit the GSK-3-mediated phosphorylation of $\beta$-catenin, stabilising it on the future dorsal side of the embryo. Previous studies indicated that FRAT1 is a component of the Wnt signalling pathway (Yost et al, 1998; Thomas et al, 1999). Overexpression of FRAT1 leads to $\beta$-catenin stabilisation through dissociation of GSK- $3 \beta$ from Axin and inhibition of $\beta$-catenin phosphorylation (Li et al, 1999; Thomas et al, 1999). Unphosphorylated $\beta$-catenin is stabilised and translocated to the nucleus to associate with TCF/LEF transcription factors (Giles et al, 2003). The $\beta$-catenin/TCF complex activates the transcription of Wnt target genes such as $c-m y c$ (He et al, 1998), cyclin D1 (Tetsu and McCormick, 1999) and human pituitary tumour transforming gene ( $h P T T G$ ) (Zhou et al, 2005). These genes play an important role in the development and formation of some neoplasias. Thus, FRAT1 is a positive regulator of the Wnt $/ \beta$-catenin pathway. Since the identification of human FRAT1, an additional homologue has been discovered in the human genome. FRAT2, just like FRAT1, functions as positive regulator of the Wnt pathway. FRAT1 and FRAT2 are more homologous in the $\mathrm{N}$-terminal region, but are completely divergent in the C-terminal region (Saitoh et al, 2001; Freemantle et al, 2002). So our probes against human FRAT1 span the C-terminal coding region of this gene, minimising the potential for crossreactivity between human FRAT1 and FRAT2. Recently, results from van Amerongen et al (2005) and van Amerongen and Berns (2005) demonstrate that murine Frat is not an essential component of the canonical Wnt pathway in mice. However, since that the regulatory mechanisms about Wnt pathway are complex and still obscure, there may be the presence of parallel pathways that are functionally redundant and may compensate for the loss of Frat. Furthermore, it is notable that some previous reports suggest that Frat plays a role in tumour progression (Jonkers et al, 1997, 1999). Therefore, it implies that in abnormal conditions, such as in the FRAT-overexpressed cancer cells, FRAT may be an important molecular event that might lead to the aberrant activation of Wnt pathway.

In this study, we used in situ hybridisation probes to demonstrate the presence of FRAT1 in paraffin-embedded human normal tissues and ovarian cancer tissues. To our knowledge, no other research group has been able to produce in situ hybridisation probes against FRAT1 to date. In situ hybridisation seems to be a more selective and more specific technique than immunohistochemistry, but requires greater care in preparing nucleasefree solutions. Tissue microarrays were also used for the rapid and efficient analysis of large numbers of paraffin-embedded tissues (Braunschweig et al, 2004, 2005). By using in situ hybridisation to detect the expression of FRAT1 and by using immunohistochemistry to detect the expression of $\beta$-catenin on tissue microarrays of paraffin-embedded ovarian adenocarcinomas, we report here that cytoplasmic/nuclear accumulation of $\beta$-catenin is closely associated with FRAT1 overexpression in ovarian serous adenocarcinomas. Although it is uncertain whether FRAT1 expression is the cause or effect of cytoplasmic $\beta$-catenin in certain circumstances, until now, almost all data shown in the literatures indicate that FRAT1 is a positive regulator of the $\mathrm{Wnt} / \beta$-catenin pathway. No reports now suggest that $\mathrm{Wnt} / \beta$-catenin pathway can regulate the expression of FRAT1. Thus, a strong correlation between expression of FRAT1 and the subcellular localisation of $\beta$-catenin indicates that FRAT1 may modulate the $\mathrm{Wnt} / \beta$-catenin pathway in ovarian serous adenocarcinomas. A study with larger cohort seems mandatory to clarify whether the same role of FRAT1 exists in other histological subtypes of ovarian cancer, such as clear cell, endometrioid and mucinous adenocarcinomas. In addition, we report that FRAT1 is detected in some normal human tissues, including endometrium, testis, pancreas and prostate. Similar results have been obtained by other researchers (Freemantle et al, 2002). Unfortunately, normal human ovary tissues are not included in this tissue microarray. However, data from Freemantle et al (2002) demonstrated that human FRAT1 expression levels in the ovary were relatively low but detectable. van Amerongen and Berns (2005) suggest that murine Frat is expressed in rapidly dividing cells (van Amerongen et al, 2005). Similar expression pattern of human FRAT1 may also be observed, but it needs further investigation.

In conclusion, FRAT1 mRNA and $\beta$-catenin protein expression were studied in 60 ovarian adenocarcinomas, including 41 serous, 11 clear cell, five endometrioid and three mucinous adenocarcinomas. We report the expression pattern of FRAT1 in some normal human tissues and that overexpression of FRAT1 in ovarian serous adenocarcinomas is significantly associated with cytoplasmic/nuclear accumulation of $\beta$-catenin. $\mathrm{Wnt} / \beta$-catenin signalling may be aberrantly activated through FRAT1 overexpression in ovarian serous adenocarcinomas. The mechanism behind the overexpression of FRAT1 in ovarian serous adenocarcinomas and its significance is yet to be investigated.

\section{ACKNOWLEDGEMENTS}

We thank Dr Liyan Xue and Dr Ning Lu for pathological support. This work was supported by National Natural Science Foundation (39925020, 30271451), National Basic Research Program (G1998051204, 2004CB518701), PR China and in part by the Intramural Research Program of the NIH, National Cancer Institute, Center for Cancer Research, US.

\section{REFERENCES}

Bell DA (2005) Origins and molecular pathology of ovarian cancer. Mod Pathol 18(Suppl 2): S19-S32

Braunschweig T, Chung JY, Hewitt SM (2004) Perspectives in tissue microarrays. Comb Chem High Throughput Screen 7: 575-585

Braunschweig T, Chung JY, Hewitt SM (2005) Tissue microarrays: bridging the gap between research and the clinic. Expert Rev Proteomics 2: 325336

Ferkey DM, Kimelman D (2002) Glycogen synthase kinase-3 beta mutagenesis identifies a common binding domain for GBP and Axin. $J$ Biol Chem 277: 16147-16152

Fraser E, Young N, Dajani R, Franca-Koh J, Ryves J, Williams RS, Yeo M, Webster MT, Richardson C, Smalley MJ, Pearl LH, Harwood A, Dale TC
(2002) Identification of the Axin and Frat binding region of glycogen synthase kinase-3. J Biol Chem 277: 2176-2185

Freemantle SJ, Portland HB, Ewings K, Dmitrovsky F, DiPetrillo K, Spinella MJ, Dmitrovsky E (2002) Characterization and tissue-specific expression of human GSK-3-binding proteins FRAT1 and FRAT2. Gene 291: $17-27$

Gamallo C, Palacios J, Moreno G, Calvo de MJ, Suarez A, Armas A (1999) Beta-catenin expression pattern in stage I and II ovarian carcinomas: relationship with beta-catenin gene mutations, clinicopathological features, and clinical outcome. Am J Pathol 155: 527-536

Giles RH, van Es JH, Clevers H (2003) Caught up in a Wnt storm: Wnt signaling in cancer. Biochim Biophys Acta 1653: 1-24 
He TC, Sparks AB, Rago C, Hermeking H, Zawel L, da Costa LT, Morin PJ, Vogelstein B, Kinzler KW (1998) Identification of c-MYC as a target of the APC pathway. Science 281: 1509-1512

Hewitt SM (2004) Design, construction, and use of tissue microarrays. Methods Mol Biol 264: $61-72$

Jonkers J, Korswagen HC, Acton D, Breuer M, Berns A (1997) Activation of a novel proto-oncogene, Frat1, contributes to progression of mouse T-cell lymphomas. EMBO J 16: $441-450$

Jonkers J, Weening JJ, van der Valk M, Bobeldijk R, Berns A (1999) Overexpression of Frat1 in transgenic mice leads to glomerulosclerosis and nephrotic syndrome, and provides direct evidence for the involvement of Frat1 in lymphoma progression. Oncogene 28: 5982 - 5990

Karbova E, Davidson B, Metodiev K, Trope CG, Nesland JM (2002) Adenomatous polyposis coli (APC) protein expression in primary and metastatic serous ovarian carcinoma. Int J Surg Pathol 10: 175-180

Kildal W, Risberg B, Abeler VM, Kristensen GB, Sudbo J, Nesland JM, Danielsen HE (2005) Beta-catenin expression, DNA ploidy and clinicopathological features in ovarian cancer: a study in 253 patients. Eur J Cancer 41: 1127 - 1134

Li L, Yuan H, Weaver CD, Mao J, Farr III GH, Sussman DJ, Jonkers J, Kimelman D, Wu D (1999) Axin and Fratl interact with dvl and GSK, bridging Dvl to GSK in Wnt-mediated regulation of LEF-1. EMBO J 18: $4233-4240$

Nelson WJ, Nusse R (2004) Convergence of Wnt, beta-catenin, and cadherin pathways. Science 303: 1483-1487

Parkin DM, Bray F, Ferlay J, Pisani P (2005) Global cancer statistics, 2002. CA Cancer J Clin 55: 74- 108

Rask K, Nilsson A, Brannstrom M, Carlsson P, Hellberg P, Janson PO, Hedin L, Sundfeldt K (2003) Wnt-signalling pathway in ovarian epithelial tumours: increased expression of beta-catenin and GSK3beta. Br J Cancer 89: $1298-1304$

Saitoh T, Katoh M (2001) FRAT1 and FRAT2, clustered in human chromosome $10 \mathrm{q} 24.1$ region, are up-regulated in gastric cancer. Int $J$ Oncol 19: $311-315$

Saitoh T, Mine T, Katoh M (2002) Molecular cloning and expression of proto-oncogene FRAT1 in human cancer. Int J Oncol 20: 785-789

Saitoh T, Moriwaki J, Koike J, Takagi A, Miwa T, Shiokawa K, Katoh M (2001) Molecular cloning and characterization of FRAT2, encoding a positive regulator of the WNT signaling pathway. Biochem Biophys Res Commun 281: 815-820

Takada T, Yagi Y, Maekita T, Imura M, Nakagawa S, Tsao SW, Miyamoto K, Yoshino O, Yasugi T, Taketani Y, Ushijima T (2004) Methylationassociated silencing of the Wnt antagonist SFRP1 gene in human ovarian cancers. Cancer Sci 95: 741-744

Tetsu O, McCormick F (1999) Beta-catenin regulates expression of cyclin D1 in colon carcinoma cells. Nature 398: $422-426$

Thomas GM, Frame S, Goedert M, Nathke I, Polakis P, Cohen P (1999) A GSK3-binding peptide from FRAT1 selectively inhibits the GSK3catalysed phosphorylation of axin and beta-catenin. FEBS Lett 458: $247-251$

Ueda M, Gemmill RM, West J, Winn R, Sugita M, Tanaka N, Ueki M, Drabkin HA (2001) Mutations of the beta- and gamma-catenin genes are uncommon in human lung, breast, kidney, cervical and ovarian carcinomas. Br J Cancer 85: 64-68

van Amerongen R, Berns A (2005) Re-evaluating the role of Frat in Wntsignal transduction. Cell Cycle 4: 1065-1072

van Amerongen R, Nawijn M, Franca-Koh J, Zevenhoven J, van der GH, Jonkers J, Berns A (2005) Frat is dispensable for canonical Wnt signaling in mammals. Genes Dev 19: 425-430

Wang Y, Zhou X, Zhu H, Liu S, Zhou C, Zhang G, Xue L, Lu N, Quan L, Bai J, Zhan Q, Xu N (2005) Overexpression of EB1 in human esophageal squamous cell carcinoma (ESCC) may promote cellular growth by activating beta-catenin/TCF pathway. Oncogene 24: 6637-6645

Wright K, Wilson P, Morland S, Campbell I, Walsh M, Hurst T, Ward B, Cummings M, Chenevix-Trench G (1999) Beta-catenin mutation and expression analysis in ovarian cancer: exon 3 mutations and nuclear translocation in $16 \%$ of endometrioid tumours. Int $J$ Cancer 82: $625-629$

Yost C, Farr III GH, Pierce SB, Ferkey DM, Chen MM, Kimelman D (1998) GBP, an inhibitor of GSK-3, is implicated in Xenopus development and oncogenesis. Cell 93: 1031-1041

Zhou C, Liu S, Zhou X, Xue L, Quan L, Lu N, Zhang G, Bai J, Wang Y, Liu Z, Zhan Q, Zhu H, Xu N (2005) Overexpression of human pituitary tumor transforming gene (hPTTG), is regulated by beta-catenin/TCF pathway in human esophageal squamous cell carcinoma. Int J Cancer 113: $891-898$ 